\title{
A Low In-Band Radiation Superregenerative Oscillator
}

\author{
Pere Palà-Schönwälder, Member, IEEE, Jordi Bonet-Dalmau, Member, IEEE, F. Xavier Moncunill-Geniz, \\ Francisco del Águila-López and Rosa Giralt-Mas
}

\begin{abstract}
This paper describes a superregenerative voltagecontrolled oscillator as a building block for superregenerative receivers where most of the oscillator spectrum components are outside the reception frequency band. This allows to overcome one of the main drawbacks of superregenerative receivers, namely the potential interference to nearby receivers operating at the same frequency due to oscillator reradiation. We perform a qualitative analysis of the solution of the circuit equations, describe the most relevant parameters for design and provide some numerical simulation results. Experimental results on a proof-ofconcept implementation validating the described principle and a discussion of the observed behavior are provided.
\end{abstract}

Index Terms-Radio receiver, superregenerative receiver, oscillator reradiation, low power.

\section{INTRODUCTION}

$\mathbf{T}$ HE superregenerative (SR) receiver [1], [2] has proven to be a suitable architecture for low-cost and low-power wireless data links. This is due to a significantly reduced complexity, especially in comparison with conventional receivers. This receiver has been traditionally used in remote control systems, short distance telemetry and wireless security, where the drawbacks of this receiver, mainly its excessive reception bandwidth in comparison with conventional receivers, are more than compensated by the reduction in complexity. In the last years, the SR receiver has been revisited and several integrated implementations have been reported [3]-[5], some of its drawbacks have been eliminated and its field of application has been broadened, with papers reporting applications to spread-spectrum [6] and UWB communications [7], [8]. On the other hand, applications of these receivers beyond classic ASK modulation have been reported [9], [10] and the SR principle has even been extended to broadband amplifiers and mixers [11].

The design of SR receivers intended to detect signals at a carrier frequency $f$ requires paying special attention to the potential problem of oscillator reradiation, especially (although not only) through the antenna, which may interfere with nearby receivers operating at or near the same frequency. For instance, this is a significant problem when developing

Manuscript received xx, 2012; revised xx, 2012. Work supported by the Spanish Dirección General de Investigación under Grant TEC2009-09924.

P. Palà-Schönwälder, J. Bonet-Dalmau, F. del-Águila-López and R. GiraltMas are with the Department of Electronic System Design and Programming (DiPSE), School of Engineering of Manresa (EPSEM), Universitat Politècnica de Catalunya (UPC), 08242 Manresa, Spain (e-mail: pere.pala@upc.edu). F. Xavier Moncunill-Geniz is with the Department of Signal Theory and Communications (TSC), Universitat Politècnica de Catalunya (UPC), 08034 Barcelona, Spain.

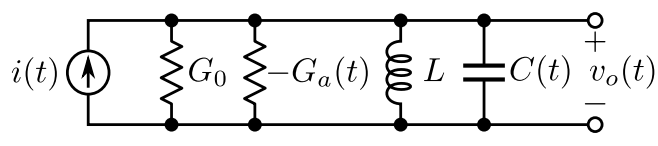

Fig. 1. Equivalent circuit of a SRO (when $C(t)$ is constant) or an SRVCO. The constant conductance $G_{0}$ includes the source resistance and the losses in the tank circuit while the periodic time-varying conductance $G_{a}(t)$ determines, among other properties, the stability or instability periods of the circuit [13].

wireless sensor networks [12], one of the currently most promising fields of application of SR receivers. This undesired phenomenon is due to the fact that the main building block of the SR receiver, the SR oscillator (SRO), generates RF bursts of appreciable amplitude at a frequency $f_{0}$ which is very close to $f$. In conventional receivers, reradiation is minimized by designing the low-noise amplifier with the additional constraint of exhibiting high reverse isolation. The required isolation may be achieved making use of a cascode structure, but this places a lower limit on the power supply voltage. Other alternatives, such as making use of two cascaded amplifier stages, also result in increased power consumption.

In this paper we propose a new idea to overcome the problem of oscillator reradiation. We design a SR voltagecontrolled oscillator (SRVCO) and provide a control signal such that the SRVCO frequency is $f$ during the sensitivity period of the quench cycle. Once the oscillator amplitude starts growing, the oscillator frequency is increased (or decreased). The envelope of the generated waveform carries the required information for signal demodulation (as in a conventional SR receiver) while the signal energy is shifted to frequencies higher (or lower) than $f$, strongly reducing the interference to other receivers operating at $f$. The spectral energy shifting effect combined with the bandpass responses of the matching circuits and the antenna, which are all typically narrowband around $f$, contribute to significantly reducing the restrictions on isolation of the low-noise amplifier.

\section{Voltage-Controlled Superregenerative OSCILLATOR}

Figure 1 depicts an equivalent circuit of several possible implementations of an SRO (if $C(t)$ is constant) or an SRVCO (if $C(t)$ is time-varying). This circuit is described by the differential equation

$$
\begin{aligned}
& \ddot{v}_{o}(t)+\frac{2 \dot{C}(t)+G_{0}-G_{a}(t)}{C(t)} \dot{v}_{o}(t)+ \\
& \frac{\frac{1}{L}+\ddot{C}(t)-\dot{G}_{a}(t)}{C(t)} v_{o}(t)=\frac{1}{C(t)} \dot{i}(t) .
\end{aligned}
$$




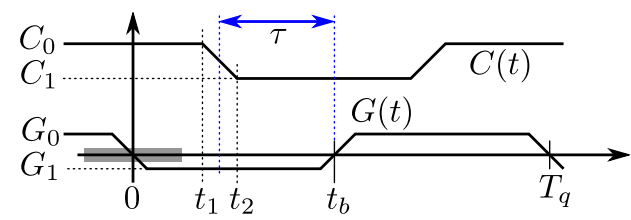

Fig. 2. Waveforms of $C(t)$ and $G(t)$. The shaded area depicts the sensitivity period.

In the linear mode of operation, the response of a classical SRO with constant capacitance value, $C(t)=C_{0}$, to an input signal described by

$$
i(t)=I p_{c}(t) \cos (\omega t+\phi),
$$

where $\omega=2 \pi f, \phi$ is a constant phase term, and $p_{c}(t)$ is a normalized pulse envelope, may be approximately written as [13]

$$
v_{o}(t)=I K|H(\omega)| p(t) \cos \left(\omega_{0} t+\phi+\angle H(\omega)\right),
$$

where $H(\omega)$ is a bandpass function centered at $\omega_{0}=2 \pi f_{0}$ and the unity normalized oscillation envelope $p(t)$ is given by

$$
p(t)=e^{-\omega_{0} \int_{t_{b}}^{t} \zeta(\lambda) d \lambda}
$$

with

$$
\omega_{0}=1 / \sqrt{L C_{0}}
$$

and

$$
\zeta(t)=\frac{G_{0}}{2 C_{0} \omega_{0}}\left(1-\frac{G_{a}(t)}{G_{0}}\right) .
$$

From (3) it follows that the oscillator signal $v_{o}(t)$ has a strong frequency content around $\omega_{0}$ during the whole SRO operation. In normal operation conditions, $\omega \approx \omega_{0}$. On the other hand, the peak gain $K$ of the superregenerative oscillator, which depends on several factors (most importantly, the time $t_{b}$ during which the circuit is unstable), is usually sufficiently high to produce an output signal $v_{o}(t)$ with peak amplitudes of some hundreds of millivolts. These relatively high signal levels in the close vicinity of the input frequency $\omega$ may be reradiated, interfering with nearby receivers.

Furthermore, in the classical SRO, $v_{o}(t)$ is only influenced by the input signal during a short time interval, the sensitivity period, centered around the instant where the net conductance $G(t)=G_{0}-G_{a}(t)$ of the circuit becomes negative [13], which is taken as $t=0$ in Fig. 2. The signal generated during the sensitivity period together with the circuit parameters determine the future evolution of the circuit variables, meaning that there is no dependence on the input signal outside the sensitivity period.

The solution of (1) for arbitrary variations of $G_{a}(t)$ and $C(t)$ is not trivial and is beyond the scope of the current paper. However, to gain insight into the circuit operation we may consider the waveforms depicted in Fig. 2, where $C(t)$ varies between $C_{0}$ and $C_{1}$ and $G$ varies between $G_{0}$ and $G_{1}$.

Figure 3 depicts the results of the numerical integration of the circuit equations taking normalized values $L=1, C_{0}=1$, $C_{1}=0.8, G_{0}=0.07, G_{1}=-0.02$ and a sinusoidal input signal with $I=10 \mu A$ and $\omega=1$, which correspond to reasonable values of a real design conveniently normalized.

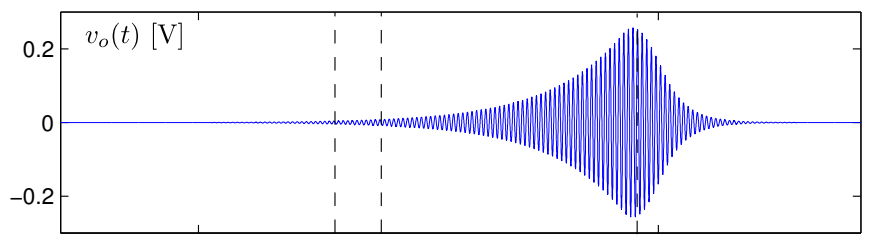

(a)

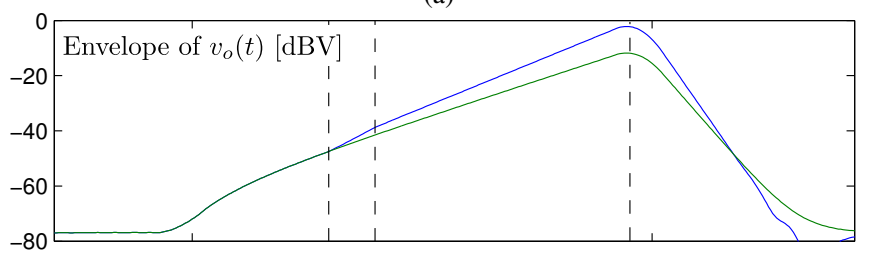

(b)

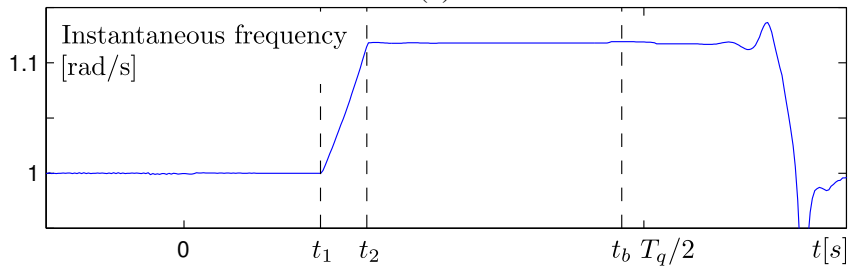

(c)

Fig. 3. Simulation results. 3a) SRVCO waveform $v_{o}(t)$ [V] for constant $C(t)=C_{0}$. 3b) The envelope of $v_{o}(t)$ in $\mathrm{dBV}$ for constant $C(t)=C_{0}$ (bottom) and time-varying $C(t)$ (top). 3c) Instantaneous pulsation of $v_{o}(t)$.

$C(t)$ and $G(t)$ have a duty cycle of $50 \%$ and exhibit rise and fall times which are $5 \%$ of the quench period. Figure $3 \mathrm{a}$ shows the generated signal $v_{o}(t)$, Fig. $3 \mathrm{~b}$ shows the envelope of $v_{o}(t)$, expressed in $\mathrm{dBV}$ to explicitly show the exponential dependence with time, and Fig. $3 \mathrm{c}$ shows the instantaneous frequency of $v_{o}(t)$, computed from the zero crossings of $v_{o}(t)$. Next, we discuss the observed behavior.

Considering that the signal from the previous quench cycle is negligible, the output signal up to $t=t_{1}$ (see Fig. 2 and Fig. 3) corresponds to that of a conventional SRO with constant capacitance $C(t)=C_{0}$ with the normalized oscillation envelope, $p(t)$, given by (4). In this interval, the instantaneous frequency is constant and given by (5).

Note also that if $t_{1}$ is outside the sensitivity period, the evolution for $t>t_{1}$ is essentially independent of the input signal. As a consequence, the overall frequency dependence of the SRVCO will be determined by the frequency dependence during the sensitivity period, which is given by $|H(\omega)|$, as in [13], with the center frequency given by (5).

In the time interval $t_{2} \leq t \leq t_{b}, C(t)=C_{1}$. As a consequence, the SRVCO signal is expected to have an envelope similar to (4) substituting $C_{0}$ by $C_{1}$ in (5) and (6). Hence, most of the SRVCO signal energy will be located around

$$
\omega_{1}=1 / \sqrt{L C_{1}}
$$

which is the value of the instantaneous frequency in this interval.

Note that, since $C_{1}<C_{0}$, the slope in this interval (Fig. $3 \mathrm{~b})$ is higher than the slope for $t<t_{1}$, producing a higher total gain. Choosing $C_{1}>C_{0}$ the SRVCO would exhibit a lower gain than with fixed $C(t)=C_{0}$ and most of the signal energy would be at frequencies lower than $\omega$. The extra gain 


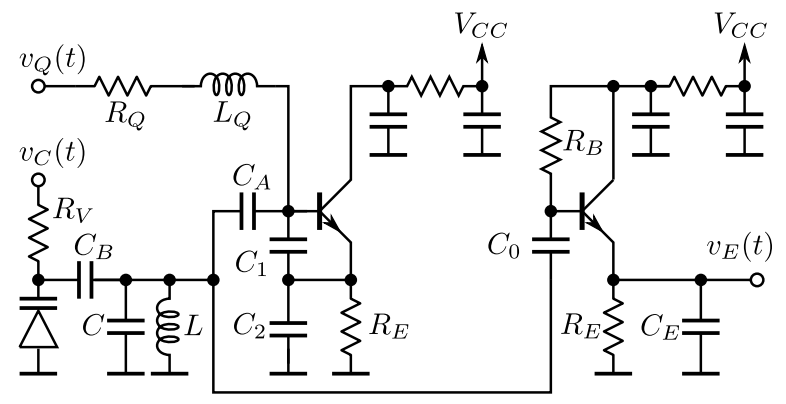

Fig. 4. Schematic of the implemented SRVCO and envelope detector. $V_{Q}(t)$ : quench signal. $V_{C}(t)$ : VCO control signal. $V_{E}(t)$ : envelope detector output.

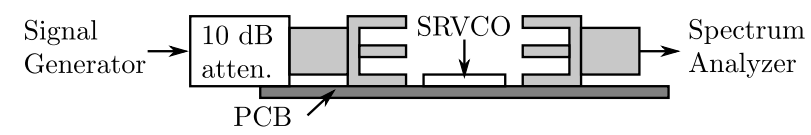

Fig. 5. RF input and output signal coupling.

achieved may be a good reason to choose $C_{1}<C_{0}$.

During the time interval $t_{1} \leq t \leq t_{2}$ the SRVCO output frequency (Fig. 3c) changes as in a conventional VCO, since the rate of change of $C(t)$ is slow compared to the generated frequency. A soft transition is observed in the SRVCO envelope during this interval.

\section{EXPERIMENTAL RESULTS AND DISCUSSION}

To validate these ideas, a proof-of-concept SRVCO in the $800 \mathrm{MHz}$ European ISM band has been built with discrete components on an FR4 board following the schematic depicted in Fig. 4. As may be seen, the SRVCO is based on a commoncollector Colpitts oscillator based on a BFP405 transistor with a BB131 varicap diode controlling the resonant frequency of the selective network. Component values are $R_{V}=5.6 \mathrm{k} \Omega$, $C_{B}=2.5 \mathrm{pF}, C=0.47 \mathrm{pF}, L=5.6 \mathrm{nH}, C_{A}=1.5 \mathrm{pF}$, $C_{1}=2.7 \mathrm{pF}, C_{2}=1 \mathrm{pF}$. The envelope detector is also built with a BFP405 transistor and allows further signal processing and final bit decision in other stages. Component values are $C_{0}=12 \mathrm{pF}, R_{B}=750 \mathrm{k} \Omega$ and $C_{E}=82 \mathrm{pF} . R_{E}=1.8 \mathrm{k} \Omega$ in both circuits and the $V_{C C}=3.3 \mathrm{~V}$ power supply is provided through conventional bypass and filtering elements.

Since we wished to investigate the inherent performance of the SRVCO concept, no previous amplifier stage was provided. Also, as the objective is to make repeatable measurements, but there is no need to measure absolute sensitivity figures (there is no reason to expect differences with previously reported ones), we have used the simple ad-hoc test setup depicted schematically in Fig. 5. The RF input signal and the output to the spectrum analyzer are loosely coupled to the SRVCO making use of MCX connectors soldered on the ground plane closely to the SRVCO with no direct connection to the circuit and using the center pins of the connectors as small antennas.

Figure 6 shows the relevant signal waveforms of the prototype. The top trace (trace \#2) is the envelope detector output showing the receiver operating at the threshold of the logarithmic mode. The varicap control signal is the center trace (trace \#3) in this figure. The adjustment shown places

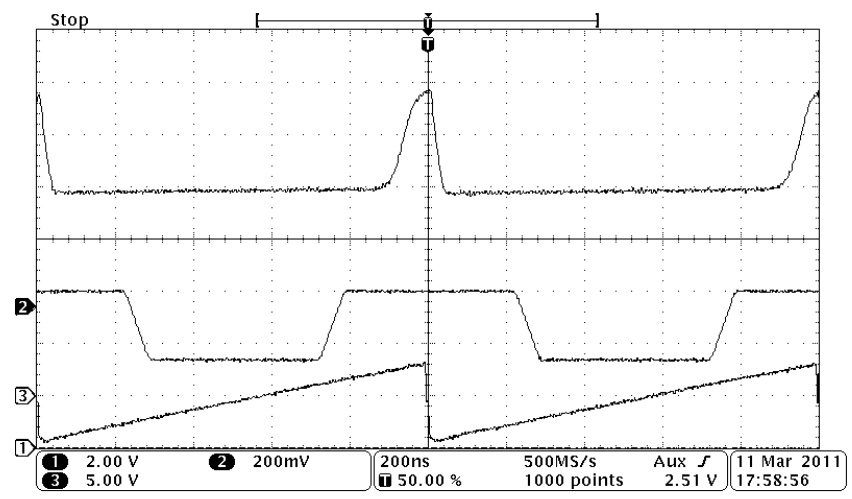

Fig. 6. SRVCO waveforms. Top: envelope detector output. Center: Varicap control signal. Bottom: Quench signal.

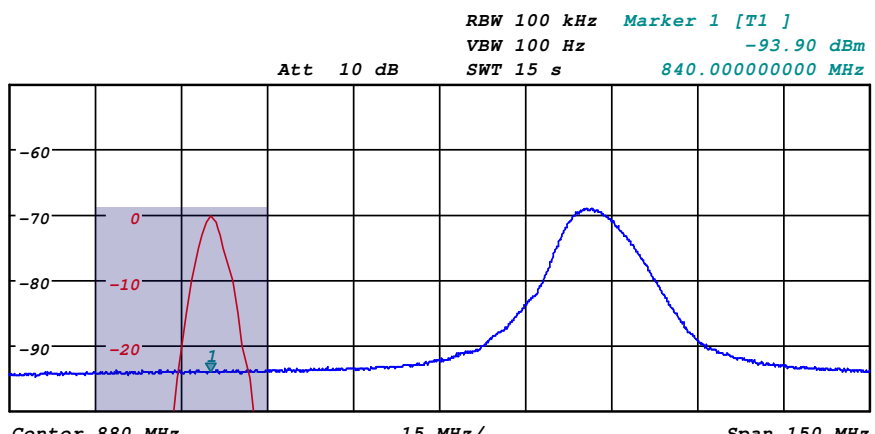

(a)

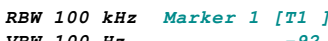
VBW $100 \mathrm{~Hz} \quad-92.97 \mathrm{dBm}$

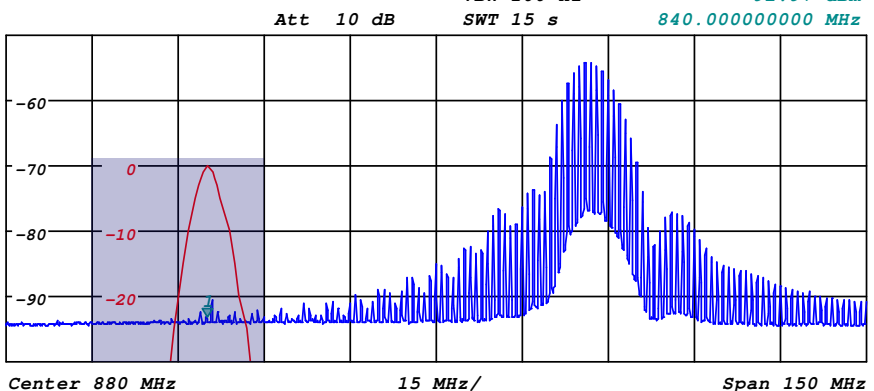

(b)

Fig. 7. Measured spectra for an SRVCO tuned to $840 \mathrm{MHz}$ (indicated by the marker and by the superimposed resulting frequency response) and providing a frequency shift of $66 \mathrm{MHz}$. 7a) Spectrum in the absence of signal. 7b) Spectrum in the presence of a CW signal at $840 \mathrm{MHz}$.

the center of the receiver sensitive passband at $840 \mathrm{MHz}$ and provides a maximum frequency shift of $+66 \mathrm{MHz}$. The bottom trace (trace \#1) depicts the applied quench voltage $v_{Q}(t)$. This signal is inversely related to the conductance $G(t)$ : The circuit is stable $(G(t)>0)$ for low values of the quench signal. For this experiment a sawtooth quench signal was chosen, resulting in a narrower receiver bandwidth, as predicted by conventional theory [2], [13]. The quench frequency is $1 \mathrm{MHz}$, which is within the modulation bandwidth of the VCO.

The observed behavior closely matches the predictions. As, in this case $C_{1}<C_{0}$, the peak gain increases with decreasing $C_{1}$, i.e. with increasing varicap control voltage.

Figure 7 shows the measured signal spectrum when the SRVCO is tuned to $840 \mathrm{MHz}$, as indicated by the markers and the superimposed frequency response of the receiver. The 
subfigures depict the signal generated by the $\operatorname{SRVCO} a$ ) in the absence of input signal (noise-driven response) and $b$ ) in the presence of a $\mathrm{CW}$ signal at $840 \mathrm{MHz}$. These spectra exhibit the characteristic shape of a SR receiver tuned to $840 \mathrm{MHz}$ but with the spectral peaks shifted upwards approximately $+66 \mathrm{MHz}$. Note that the in-band radiated power density is attenuated by more than $35 \mathrm{~dB}$ (see Fig. 7b) compared to what would be obtained with a classical SRO where the peak located at $906 \mathrm{MHz}$ would be at $840 \mathrm{MHz}$. On the other hand, the resulting out-of-band attenuation will be strongly dependent on several factors which will be unique to each design: the antenna $Q$, the antenna matching circuit bandwidth, the combined frequency response of the LNA and the corresponding LNA-VCSRO coupling circuit and, most important, the particular relative frequency shift $\alpha=\omega_{1} / \omega_{0}$. Assuming $\alpha=1.08$ and moderate values of $Q$ (in the range of 10 to 15 ) in each of these bandpass terms, the combined response achieves $\sim 20 \mathrm{~dB}$ of attenuation to be added to the reverse isolation of the LNA.

The measured receiver frequency response also matches that of a conventional receiver, as expected. However, as the time point $t_{1}$ (where the capacitance starts changing) is decreased (which may be attractive to achieve more overall gain), an increasing part of the sensitivity period includes a zone where the receiver is sensitive to higher frequencies. So, the shape of the frequency response also gives a useful hint to indirectly determine the end of the sensitivity period.

An experimental demonstration emphasizing this effect is presented in Fig. 8a and Fig. 8b, which correspond to an SRVCO adjusted to provide $+33 \mathrm{MHz}$ and $+66 \mathrm{MHz}$ shifts in the spectral peak, respectively. In these figures we show the measured frequency responses for three different relative phase shifts between $C(t)$ and $G(t)$. There are steps of $10^{\circ}$ phase shift, corresponding to $27.8 \mathrm{~ns}$, between traces. As the figure caption shows, we have referenced these traces to $t_{b}$ instead of the origin, as $t_{b}$ is much better determined for the sawtooth quench used in the experiments.

A detailed analysis of the resulting frequency response is still open to current research and will be investigated in detail elsewhere but the qualitative effect corresponds to the qualitative predictions outlined before.

\section{CONCLUSION}

In this letter we have presented a superregenerative voltagecontrolled oscillator. We have shown how the simultaneous control of the quench signal and the oscillation instantaneous frequency allows retaining the essential properties of a conventional SR receiver with the advantageous ability to modulate the locally generated RF signal away from the reception frequency. Experimental results on a $800 \mathrm{MHz}$ ISMband receiver validate the proposed approach, demonstrating a significant reduction of in-band radiated power, lowering the interference to nearby receivers in the same frequency band. The choice of capacitance variation influences the envelope of the generated RF bursts and may also be exploited to achieve a higher gain compared to a SR receiver operating at a fixed frequency. The exact investigation of the effects

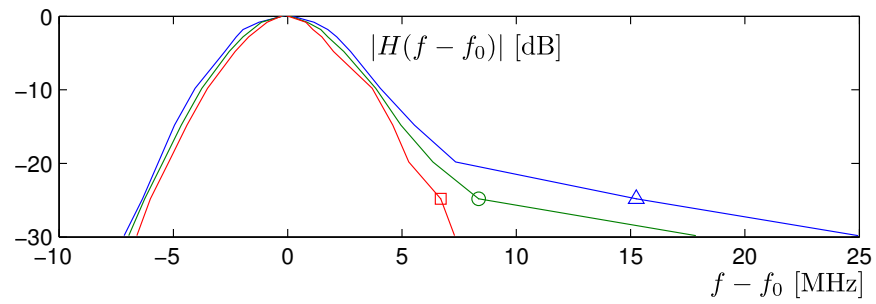

(a)

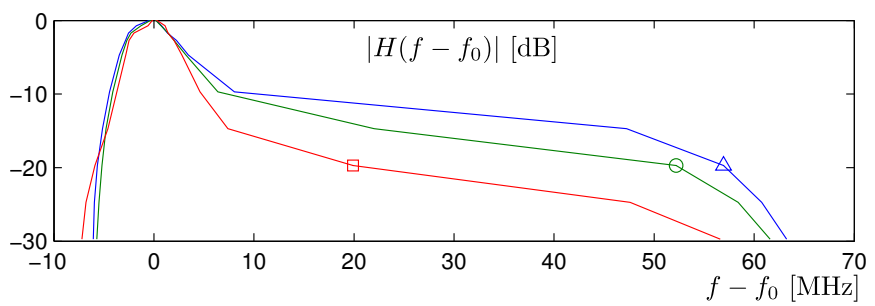

(b)

Fig. 8. Measured frequency responses for an SRVCO operating with $a$ ) $+33 \mathrm{MHz}$ frequency shift, $b)+66 \mathrm{MHz}$ frequency shift. $\square: \tau=175 \mathrm{~ns}$, o: $\tau=203$ ns, $\triangle: \tau=230$ ns, with $\tau=t b-\left(t_{1}+t_{2}\right) / 2$ (see also Fig. 2 ).

of arbitrary variations of the control signal of the voltagecontrolled oscillator and the development of design guidelines to meet specific criteria are still open areas for further research.

\section{REFERENCES}

[1] E. H. Armstrong, "Some recent developments of regenerative circuits," in Proc. IRE, Aug. 1922, vol. 10, pp. 244-260.

[2] J. R. Whitehead, Super-Regenerative Receivers. Cambridge, U.K.: Cambridge Univ. Press, 1950.

[3] M. Pelissier, J. Jantunen, B. Gomez, J. Arponen, G. Masson, S. Dia, J. Varteva, and M. Gary, "A $112 \mathrm{Mb} / \mathrm{s}$ full duplex remotely-powered im- pulse-UWB RFID transceiver for wireless NV-memory applications," IEEE J. Solid-State Circuits, vol. 46, no. 4, pp. 916-927, Apr. 2011.

[4] Vouilloz, A.; Declercq, M.; Dehollain, C., "A low-power CMOS superregenerative receiver at $1 \mathrm{GHz}$," Solid-State Circuits, IEEE Journal of , vol.36, no.3, pp.440-451, Mar 2001

[5] Jia-Yi Chen; Flynn, M.P.; Hayes, J.P., "A Fully Integrated Auto-Calibrated Super-Regenerative Receiver in 0.13- $\mu \mathrm{m}$ CMOS," Solid-State Circuits, IEEE Journal of , vol.42, no.9, pp.1976-1985, Sept. 2007

[6] F. Xavier Moncunill-Geniz, P. Palà-Schönwälder, C. Dehollain, N. Joehl and M. Declercq, "A 2.4-GHz DSSS superregenerative receiver With a Simple Delay-Locked Loop," IEEE Microw.Wireless Comp. Letters, vol. 15, no. 8, 2005, pp. 499-501.

[7] M. Anis, R. Tielert, N. Wehn, "Low power complementary-Colpitts selfquenched super-regenerative ultra-wideband (UWB) bandpass filter in CMOS technology," in Proc. IEEE MTT-S Micr. Symp. Dig., 2008, pp. 1047-1049.

[8] F. Xavier Moncunill-Geniz, J. Bonet-Dalmau, P. Palà-Schönwälder, F. delÁguila-López and R. Giralt-Mas, "A High-Resolution UWB IR Superregenerative Receiver Front End With an SRD Quench Shaper," in IEEE Trans. Circuits Syst. II, Exp. Briefs, vol. 59, no. 2, pp. 93-97, Feb. 2012.

[9] L. Hernandez, S. Paton, "A superregenerative receiver for phase and frequency modulated carriers," in Proc. IEEE ISCAS, 2002, pp. III-81 - III-84.

[10] P. Palà-Schönwälder, F. Xavier Moncunill-Geniz, J. Bonet-Dalmau, F. del-Águila-López and R. Giralt-Mas, "A BPSK superregenerative receiver: Preliminary results," in Proc. IEEE ISCAS, 2009, pp. 1537-1540.

[11] P. Palà-Schönwälder, F. Xavier Moncunill-Geniz, J. Bonet-Dalmau, F. del-Águila-López and R. Giralt-Mas, "Baseband superregenerative amplification," IEEE Trans. Circuits Syst. I, Reg. Papers, vol. 56, no. 9, pp. 1930-1937, Sep. 2009.

[12] Ayers, J.; Mayaram, K.; Fiez, T.S.; , "An Ultralow-Power Receiver for Wireless Sensor Networks," Solid-State Circuits, IEEE Journal of , vol.45, no.9, pp.1759-1769, Sept. 2010

[13] F. Xavier Moncunill-Geniz, P. Palà-Schönwälder and O. Mas-Casals, "A generic approach to the theory of superregenerative reception," IEEE Trans. Circuits Syst. I, Reg. Papers, vol. 52, no. 1, pp. 54-70, Jan. 2005. 